\title{
Antecipação da diagnose foliar em arroz de sequeiro
}

\author{
Denis Borges Tomio(1), Marley Marico Utumi(2), Daniel Vidal Perez ${ }^{(3)}$, Jairo Rafael Machado Dias ${ }^{(4)}$ \\ e Paulo Guilherme Salvador Wadt( ${ }^{(5)}$
}

\begin{abstract}
(1)Universidade Federal do Acre, Campus Universitário, BR 364, Km 04, Caixa Postal 500, CEP 69915-900 Rio Branco, AC, Brasil. E-mail: denis.tomio@gmail.com ${ }^{(2)}$ Embrapa Rondônia, Caixa Postal 405, CEP 76980-000 Vilhena, RO, Brasil. E-mail: marleyutumi@hotmail.com ${ }^{(3)}$ Embrapa Solos, Rua Jardim Botânico, no 1.024, CEP 22426-000 Rio de Janeiro, RJ, Brasil. E-mail: daniel.perez@embrapa.br (4)Universidade Federal de Rondônia, Departamento de Agronomia, Avenida Norte-Sul, no 7.300, Nova Morada, CEP 76940-000 Rolim de Moura, RO, Brasil. E-mail: jairorafaelmdias@hotmail.com ${ }^{(5)}$ Embrapa Acre, Rodovia BR 364, Km 5,5, Zona Rural, Caixa Postal 127, CEP 76815-800 Porto Velho, RO, Brasil. E-mail: paulogswadt@dris.com.br
\end{abstract}

Resumo - O objetivo deste trabalho foi avaliar a possibilidade de antecipar a amostragem foliar em arroz de sequeiro, para viabilizar o diagnóstico nutricional em tempo hábil para intervenção. Foram coletadas 107 amostras foliares em ensaios de materiais genéticos da Embrapa Rondônia, no Estado de Rondônia, em três épocas de amostragem: antecipada, aos 30 e 40 dias após a semeadura (DAS); e padrão, aos 65 DAS. Foram obtidas as normas de diagnose da composição nutricional (CND) para cada época avaliada, as quais foram utilizadas para o diagnóstico em todas as épocas, tendo-se comparado os resultados do potencial de resposta à adubação sob duas ou três classes de suficiência nutricional, e verificado o grau de concordância entre os diagnósticos. $\mathrm{O}$ uso de duas classes de suficiência aumenta a possibilidade da antecipação da época de amostragem para diagnose foliar, o que viabilizaria o diagnóstico nutricional em tempo hábil para a correção ou a mitigação de eventuais deficiências nutricionais. A antecipação da diagnose foliar em arroz de sequeiro depende do desenvolvimento de normas de diagnose específicas para cada período amostral.

Termos para indexação: Oryza sativa, amostragem foliar, avaliação do estado nutricional, DRIS, normas CND, potencial de resposta à adubação.

\section{Anticipation of leaf diagnosis in upland rice}

Abstract - The objective of this work was to evaluate the possibility of anticipation of foliar sampling in upland rice, in order to enable nutritional diagnosis for timely intervention. One hundred and seven leaf samples were collected from genotype trials of Embrapa Rondônia, in the state of Rondônia, Brazil, in three sampling times: anticipated, at 30 and 40 days after sowing (DAS); and standard, at 65 DAS. Composition nutrient diagnosis (CND) norms were obtained for each evaluated time and used for diagnosis on all evaluation times, comparing the results from the potential response to fertilization under two or three nutritional sufficiency classes, and checking the degree of agreement between the diagnostics. The use of two sufficiency classes increases the possibility of anticipating sampling time for foliar diagnosis, which would enable timely nutritional diagnosis to correct or mitigate eventual nutritional deficiencies. The anticipation of foliar diagnosis in upland rice depends on the development of specific nutritional diagnosis norms for each sampling time.

Index terms: Oryza sativa, foliar sampling, assessment of nutritional status, DRIS, CND norms, response potential to fertilization.

\section{Introdução}

O arroz de terras altas (Oryza sativa L.) adapta-se a solos ácidos e de baixa fertilidade natural, motivo pelo qual essa cultura normalmente é utilizada como antecessora a cultivos de milho e soja (Barreto et al., 2002), ou em sistemas de integração lavoura-pecuária em áreas de pastagens em fase de recuperação (Macedo, 2009; Ferreira \& Santiago, 2012).

Em Rondônia, com o crescimento da rizicultura na última década, são cultivados 82 mil hectares de arroz, com produtividade média de 2,9 $\mathrm{Mg} \mathrm{ha}^{-1}$ (Instituto Brasileiro de Geografia e Estatística, 2013). Melhorias no controle fitossanitário, introdução de genótipos de alto potencial produtivo, manejo da adubação e plantios em rotação são os principais fatores responsáveis por esse aumento. No entanto, estima-se que a cultura tenha atingido, no Brasil, apenas $40 \%$ de seu potencial produtivo (Fageria et al., 2011).

Para o manejo da adubação, o monitoramento nutricional das lavouras, juntamente com a avaliação da fertilidade do solo, tem sido adotado em sistemas 
de produção com alta tecnologia (Fageria et al., 2009), pois contribui para redução nos custos com fertilizantes, ao possibilitar adubações mais equilibradas. Para a interpretação do estado nutricional das plantas, os métodos do nível crítico (NC) e das faixas de suficiência (FS) estão entre as ferramentas mais utilizadas para lavouras comerciais. Contudo, a aplicação desses métodos limita-se a lavouras sob manejo cultural e condições edafoclimáticas semelhantes às utilizadas no estabelecimento dos padrões de referência. Para contornar essa limitação, o sistema integrado de diagnose e recomendação (DRIS) é uma alternativa que apresenta menor dependência da calibração local de seus padrões de referência e que permite a ordenação dos nutrientes quanto ao grau de limitação nutricional (Beaufils, 1973; Sumner, 1977; Rodríguez \& Rodriguez, 2000).

Adiagnose da composição nutricional (CND) (Parent \& Dafir, 1992) utiliza índices de relações multivariadas (quociente do teor do nutriente em relação à média geométrica da composição nutricional); portanto, é estruturalmente semelhante ao DRIS e compartilha das mesmas vantagens do método. Tanto o DRIS como o CND, já foram estudados para a cultura do arroz irrigado por inundação (Guindani et al., 2009; Wadt et al., 2013). A menor dependência da calibração local levou alguns autores a preconizar que esses métodos podem ser utilizados independentemente do estádio de desenvolvimento da lavoura, conforme sugerido para a cultura do milho (Zea mays L.) por Sumner \& Beaufils (1975) e da cana-de-açúcar (Saccharum officinarum L.) por Sumner (1977). Entretanto, o uso de normas específicas para cada estádio de desenvolvimento é desejável, especialmente quando se pretende antecipar o período de monitoramento nutricional, como em estudos com cafeeiro (Coffea arabica L.) (Partelli et al., 2007) e maracujazeiro-amarelo (Passiflora edulis Sims) (Carvalho et al., 2011).

Existem normas DRIS para o arroz irrigado em trabalhos realizados nos Estados Unidos (Bell \& Kovar, 2009) e no Rio Grande do Sul (Guindani et al., 2009); porém, não há normas CND para a cultura do arroz, seja em condições de cultivo de terras altas ou de irrigação por inundação. Além disso, a antecipação do período de monitoramento pode ser importante para o manejo da adubação, por possibilitar que os ajustes no estado nutricional das lavouras sejam feitos de forma tempestiva à aplicação de fertilizantes, na própria safra em que o monitoramento foi realizado (Kurihara, 2004; Wadt \& Alvarez, 2005).
O objetivo deste trabalho foi avaliar a possibilidade de antecipar a amostragem foliar em arroz de sequeiro, para viabilizar o diagnóstico nutricional em tempo hábil para intervenção.

\section{Material e Métodos}

O experimento foi realizado em parcelas experimentais de ensaios de competição de cultivares e linhagens de arroz de sequeiro, conduzidos no campo experimental da Embrapa Rondônia (1247'23"S, $\left.60^{\circ} 05^{\prime} 42^{\prime \prime} \mathrm{W}\right)$, no Município de Vilhena, RO. A região apresenta clima tropical chuvoso, do tipo AW, segundo classificação de Köppen, com temperatura média anual de $23,9^{\circ} \mathrm{C}$ e precipitação pluvial média de $2.098 \mathrm{~mm}$, com seis meses secos bem definidos (abril a setembro). O período chuvoso apresenta precipitação média mensal de 312,4 mm e temperaturas mínima e máxima médias de 21,0 e $29,6^{\circ} \mathrm{C}$, respectivamente (Boletim climatológico de Rondônia, 2010).

O cultivo foi realizado em Latossolo VermelhoAmarelo distrófico de baixa fertilidade natural (Tabela 1) mas devidamente corrigido na safra 2011/2012, com espaçamento entre linhas de $0,36 \mathrm{~m}$ e densidade de plantio de 102 sementes por metro, ou $70 \mathrm{~kg}$ de semente por hectare. A área foi cultivada com soja nas safras 2008/2009 e 2009/2010, e ficou em pousio na safra 2010/2011. Não houve calagem antes do plantio.

Os tratamentos consistiram de oito materiais genéticos: cinco linhagens, LE 425, LE 719, LE 161, LE 834 e LE 658; e três cultivares, BRS Sertaneja, BRS Monarca e AN Cambará. Foram utilizadas parcelas experimentais com $100 \times 12 \mathrm{~m}(0,12 \mathrm{ha})$. A cultivar BRS Sertaneja foi duplicada em dois tratamentos; portanto, foram considerados nove tratamentos relativos aos oito genótipos avaliados. A semeadura ocorreu entre 8 e 13 de outubro de 2011, e a colheita, entre 28 de março e 10 de abril de 2012. Os materiais apresentaram período de emergência de 3 a 4 dias.

As amostragens foliares foram feitas entre as bordaduras e o centro das parcelas experimentais, tendo-se retirado quatro amostras compostas repetições da parte aérea do arroz, em três épocas de avaliação. As amostragens foram feitas em faixa de $5 \mathrm{~m}$, ao longo da maior extensão de cada parcela experimental $(100 \mathrm{~m})$. A primeira amostragem foi realizada aos 30 dias após a semeadura (DAS), e as duas seguintes foram realizadas, nas mesmas parcelas experimentais, 
aos 40 e 65 DAS, tendo-se obtido quatro amostras compostas por genótipo, em cada período de coleta. A última amostragem foi realizada para coincidir com o início do estádio reprodutivo do arroz, que corresponde à fase em que ao menos $50 \%$ das plantas apresentam o colar da folha-bandeira formado, representado pelo período R1 e R3 da escala de Counce et al. (2000).

Foram utilizadas quatro repetições, que resultaram em 108 amostras independentes (nove tratamentos com genótipos, três com épocas e quatro repetições). Em razão da perda de uma amostra foliar - a quarta repetição da cultivar BRS Sertaneja, no nono tratamento da amostragem aos 40 DAS -, o total de amostras ficou reduzido para 107 amostras foliares.

A produtividade nas parcelas experimentais foi determinada em três linhas de plantio, com a avaliação de $4 \mathrm{~m}$ em cada linha, com seis repetições. A produtividade foi expressa em $\mathrm{kg} \mathrm{ha}{ }^{-1}$, para o equivalente de $13 \%$ de umidade em grãos com casca.

Nas avaliações antecipadas, foram retiradas amostras compostas de 25 plantas, aos 30 DAS, e de 10 plantas, aos $40 \mathrm{DAS}$, tendo-se coletado toda a parte aérea do arroz em corte rente ao solo. $\mathrm{Na}$ avaliação na época convencional, aos $65 \mathrm{DAE}$, foram coletadas apenas as folhas-bandeiras, no total de 50 para cada amostra composta.

O material vegetal amostrado foi acondicionado em câmara fria, com temperatura, de $13^{\circ} \mathrm{C}$, e umidade, de $30 \%$, constantes, em sacos de papel devidamente identificados, em laboratório. O material foi preparado e submetido às análises quanto aos teores totais de $\mathrm{N}$, $\mathrm{P}, \mathrm{K}, \mathrm{Ca}, \mathrm{Mg}, \mathrm{B}, \mathrm{Cu}, \mathrm{Fe}, \mathrm{Mn}$ e $\mathrm{Zn}$, conforme Carmo et al. (2000).

As normas CND foram obtidas a partir do resultado da análise foliar das 107 amostras úteis, que estiveram relacionadas a produtividades médias superiores à estimada para o Estado. Portanto, a população padrão foi formada pelas plantas das 107 parcelas amostradas.

As concentrações foliares dos nutrientes foram ajustadas para uma mesma unidade $\left(\mathrm{mg} \mathrm{kg}^{-1}\right)$.

Tabela 1. Análise de fertilidade do solo do campo experimental da Embrapa Rondônia utilizado na avaliação dos genótipos de arroz (Oryza sativa), em Vilhena, RO.

\begin{tabular}{lccccccc}
\hline $\mathrm{pH}$ & $\mathrm{P}$ & $\mathrm{K}$ & $\mathrm{Ca}$ & $\mathrm{Mg}$ & $\mathrm{Al}+\mathrm{H}$ & $\mathrm{Al}$ & $\begin{array}{c}\mathrm{V} \\
\mathrm{em} \mathrm{KCL}\end{array}$ \\
\hline 5,11 & 4,70 & 0,10 & 2,42 & 0,69 & 4,41 & 0,00 & 42,13 \\
\hline
\end{tabular}

Em seguida, calculou-se o valor do complemento orgânico da biomassa foliar (valor $\mathrm{R}$, que se refere à biomassa da folha, descontados os nutrientes avaliados de sua composição), a partir da expressão: $\mathrm{R}=1.000 .000-(\mathrm{vN}+\mathrm{vP}+\mathrm{vK}+\mathrm{vCa}+\mathrm{vMg}$ $+\mathrm{vB}+\mathrm{vCu}+\mathrm{vFe}+\mathrm{vMn}+\mathrm{vZn}$ ), em que $\mathrm{R}$ é o valor do complemento, para $1.000 \mathrm{mg} \mathrm{kg}^{-1}$ de matéria seca, em relação à soma dos teores dos nutrientes $\mathrm{V}_{\mathrm{i}}$ ( $\mathrm{i}=\mathrm{N}, \mathrm{P}, \ldots, \mathrm{Zn}$ ), em $\mathrm{mg} \mathrm{kg}^{-1}$.

A média geométrica dos teores nutricionais foi obtida para cada amostra (Parent, 2011), e, em seguida, determinou-se o valor da variável multinutriente $(\mathrm{zX})$, de acordo com a expressão: $\mathrm{zX}=\ln (\mathrm{vX} / \mathrm{mGeo})$, em que $\mathrm{zX}$ representa o valor da relação multivariada entre o teor de nutriente avaliado $(\mathrm{Vx})$ e a média geométrica desses teores (mGeo). Com os valores de $\mathrm{zX}$ de cada amostra, calcularam-se a média aritmética $(\mathrm{mX})$ e o desvio-padrão (sX), para as três épocas de amostragem. Esses dois parâmetros descritivos da população padrão constituíram as normas CND preliminares para cada época de desenvolvimento do arroz de terras altas.

Os índices CND foram calculados a partir das normas, pela diferença entre o valor da relação multivariada de cada nutriente $(\mathrm{zX})$ e a média da norma, com o resultado dividido pelo desvio-padrão da norma (sX), para obtenção da diferença normalizada, como sugerido por Parent (2011): I X $=(z X-m X) / s X$, em que I_X representa o índice CND; $\mathrm{mX}$ representa a norma média; e sX representa o desvio-padrão da norma, para cada um dos nutrientes avaliados.

O somatório, em módulo, dos índices CND para os nutrientes de cada amostra, em cada estádio de desenvolvimento avaliado, constituiu o índice de balanço nutricional (IBN), que, dividido pelo número de nutrientes avaliados, consiste no IBN médio (IBNm). Os índices CND foram interpretados de acordo com duas ou três classes de suficiência nutricional, com base no critério do potencial de resposta à adubação (PRA) (Wadt, 2005).

Quando foram utilizadas três classes de suficiência, consideraram-se como: deficientes, os nutrientes com índice CND negativo e maior, em módulo, que o IBNm; em equilíbrio, os nutrientes com índice CND menor, em módulo, que o IBNm; e em excesso, os nutrientes com índice CND positivo e maior que o IBNm. Quando apenas duas classes foram utilizadas, consideraram-se como: insuficiente, o nutriente com índice CND negativo e maior, em módulo, que o 
IBNm; e suficiente, o nutriente com CND negativo e menor ou igual, em módulo, que o IBNm, ou com índice DRIS positivo. Utilizou-se a notação $(\mathrm{X} \rightarrow \mathrm{Y})$ para descrever o diagnóstico nutricional de amostras retiradas aos Y DAS, realizado a partir de normas CND obtidas para X DAS.

Para a avaliação do estado nutricional pelo método convencional da FS, determinou-se o intervalo de confiança (IC) dos teores médios em cada estádio de desenvolvimento, a partir da expressão: $\mathrm{IC}=\mathrm{mX} \pm \mathrm{t} \alpha . \mathrm{smX}$, em que $\mathrm{mX}$ e $\mathrm{smX}$ representam o teor médio e o desvio-padrão dos teores dos nutrientes, respectivamente; e t $\alpha$ é o valor de t bilateral, a $5 \%$ de probabilidade, com n-1 graus de liberdade. Considerou-se como FS a amplitude do IC, com resultados expressos em $\mathrm{g} \mathrm{kg}^{-1}$, para macronutrientes, e em $\mathrm{mg} \mathrm{kg}^{-1}$, para micronutrientes. Valores abaixo ou acima dos limites inferior e superior do IC representaram, respectivamente, teores nutricionais deficientes ou em excesso.

Os diagnósticos nutricionais foram realizados a partir de normas CND específicas e não específicas do arroz, para cada estádio de desenvolvimento, tendose ordenado os nutrientes de acordo com o grau de limitação nutricional representado pelos índices. Os diagnósticos também foram comparados entre si, tendo-se computado os casos de concordância entre as diferentes épocas de amostragem. Consideraram-se diagnósticos concordantes os casos em que as normas específicas para cada época tiveram os mesmos resultados das normas não específicas, tendo-se determinado a proporção (\%) desses diagnósticos.

\section{Resultados e Discussão}

Os genótipos apresentaram produtividades que variaram de $3.029 \mathrm{~kg} \mathrm{ha}^{-1}$, para a cultivar AN Cambará, a $5.583 \mathrm{~kg} \mathrm{ha}^{-1}$, para a cultivar BRS Sertaneja, com produtividade média entre todos os materiais de $4.508 \mathrm{~kg} \mathrm{ha}^{-1}$. Esses valores foram superiores à média estadual de $2.819 \mathrm{~kg} \mathrm{ha}^{-1}$ (Companhia Nacional de Abastecimento, 2014) e compatíveis com produções observadas em campo, em regiões ecofisiologicamente semelhantes (Fageria et al., 2011).

Os teores dos nutrientes nas amostras estiveram dentro das faixas propostas por Prado (2008), exceto para $\mathrm{K}, \mathrm{Fe}$ e $\mathrm{Mn}$, próximos das indicadas por Fageria et al. (2011), em trabalho de avaliação da marcha de absorção de nutrientes (Tabela 2).
Os teores dos nutrientes diminuíram entre as épocas de amostragem aos 30 e 40 DAS, exceto para Mg. Fageria et al. (2011) atribuíram essa diminuição ao efeito de diluição, em razão do intenso perfilhamento e do rápido aumento na matéria seca das plantas, durante esse período. Fageria et al. (2011) relataram que o K, mesmo com tendência de diminuição nos seus teores entre as épocas de amostragem, foi o nutriente com maior concentração, a partir da segunda amostragem, tendo sido superado pelo $\mathrm{N}$ na amostragem aos 19 DAS (Tabela 2). No entanto, no presente trabalho, o $\mathrm{N}$ apresentou as maiores concentrações, independentemente da época de amostragem. As faixas propostas por Prado (2008) também indicaram maior concentração de $\mathrm{N}$ e maior variação para $\mathrm{K}$, o que indica maior amplitude nos valores considerados como adequados para $\mathrm{K}$.

Os teores de Fe e Mn estiveram sempre abaixo dos obtidos por Prado (2008) e Fageria et al. (2011) (Tabela2). Segundo Prado(2008), esses micronutrientes são os mais abundantes em solos tropicais ácidos. Assim, apesar dos baixos teores foliares observados no presente trabalho, a probabilidade de deficiência desses micronutrientes nas plantas avaliadas deve ser considerada baixa, uma vez que os solos da área experimental eram ácidos.

$\mathrm{O}$ aumento verificado nos teores de $\mathrm{Ca}$ pode ser atribuído à baixa translocação do nutriente pela planta (Prado, 2008). Quanto ao N, houve adubações de cobertura com o nutriente, o que justificaria a tendência de estabilidade relativa observada em seus teores. Para $\mathrm{P}$, não ficou claro o efeito que resultou no aumento de seus teores, mas, possivelmente, ele esteve relacionado ao crescimento do sistema radicular fasciculado do arroz, o que teria aumentado a interface de contato solo-raiz e facilitado a absorção do nutriente por difusão (Marschner, 2012).

As normas CND variaram de acordo com as épocas de amostragem (Tabela 3), principalmente na comparação entre as amostragens realizadas na folha-bandeira (65 DAS) e as realizadas nas épocas antecipadas (30 e 40 DAS). A variação observada nos teores de $\mathrm{Fe}$ foi confirmada pelas normas CND. Nas duas primeiras amostragens, os coeficientes de variação para o nutriente estiveram acima de 44\%, mas diminuíram e ficaram abaixo dos $14 \%$ na amostragem durante o período padrão (65 DAS).

Pesq. agropec. bras., Brasília, v.50, n.3, p.250-258, mar. 2015 DOI: $10.1590 / \mathrm{S} 0100-204 X 2015000300009$ 
As normas CND também aumentaram ao longo dos períodos de amostragem para os nutrientes $\mathrm{N}, \mathrm{P}$, $\mathrm{Ca}$ e $\mathrm{Cu}$ (Tabela 3). A norma CND para $\mathrm{N}$ variou de 3,5 (30 DAS) e 3,3 (40 DAS) a 4,0 (65 DAS); para $\mathrm{P}$, de 0,7 (30 e $40 \mathrm{DAS})$ a 1,2 (65 DAS); e para $\mathrm{Ca}$, de 1,0 (30 e 40 DAS) a 1,6 (65 DAS). Já as normas para $\mathrm{K}$ e $\mathrm{Zn}$ diminuíram entre os períodos amostrais, na comparação entre as amostragens antecipadas e a tradicional.

A variação nas normas, entre as diferentes épocas, está relacionada à alteração nos teores dos nutrientes, mas não tem influência direta sobre a concordância dos diagnósticos (Tabela 4).
O diagnóstico realizado por normas específicas foi comparado para cada uma das épocas de amostragem $30 \rightarrow 30,40 \rightarrow 40$ e $65 \rightarrow 65(X \rightarrow Y)$, em que $X$ refere-se à época (DAS) de obtenção das normas CND utilizadas no diagnóstico; e Y refere-se à época de retirada das amostras. Constatou-se que os casos de insuficiência de macronutrientes mantiveram-se praticamente constantes (Tabela 4), nas três épocas: N, em 25, 23 e $25 \%$ dos casos, respectivamente; $\mathrm{P}$, em 20,20 e $17 \%$ dos casos; K, em 19, 20 e 19\% dos casos; Ca, em 22, 23 e $28 \%$ dos casos; e $\mathrm{Mg}$, em 22,20 e $22 \%$ dos casos. Já para os micronutrientes, a semelhança na proporção dos casos de insuficiência foi menor: Fe, em 31, 26 e

Tabela 2. Intervalo de confiança (95\%) dos teores nutricionais médios para arroz de sequeiro (Oryza sativa), em cada época de amostragem (em dias após a semeadura, DAS), e diferentes níveis críticos apontados pela literatura.

\begin{tabular}{|c|c|c|c|c|c|c|c|}
\hline \multirow[t]{2}{*}{ Nutriente } & \multicolumn{3}{|c|}{ Época de amostragem } & \multirow[t]{2}{*}{ Prado $(2008)^{(1)}$} & \multicolumn{3}{|c|}{ Fageria et al. $(2011)^{(2)}$} \\
\hline & $30 \mathrm{DAS}$ & 40 DAS & 65 DAS & & 19 DAS & $44 \mathrm{DAS}$ & $68 \mathrm{DAS}$ \\
\hline & \multicolumn{7}{|c|}{ Macronutrientes $\left(\mathrm{g} \mathrm{kg}^{-1}\right)$} \\
\hline $\mathrm{N}$ & $35,3-37,7$ & $25,0-27,1$ & $31,8-33,3$ & $27,0-35,0$ & 47,7 & 30,2 & 18,7 \\
\hline $\mathrm{P}$ & $2,2-2,4$ & $1,8-2,0$ & $1,9-2,1$ & $1,8-3,0$ & 3,6 & 1,7 & 1,1 \\
\hline $\mathrm{K}$ & $24,2-26,7$ & $20,9-23,3$ & $10,4-11,4$ & $13,0-30,0$ & 33,0 & 31,2 & 27,5 \\
\hline $\mathrm{Ca}$ & $2,8-3,1$ & $2,5-2,7$ & $2,8-3,3$ & $2,5-10,0$ & 3,3 & 3,8 & 3,1 \\
\hline \multirow[t]{2}{*}{$\underline{\mathrm{Mg}}$} & $2,9-3,2$ & $3,0-3,3$ & $1,8-2,0$ & $1,5-5,0$ & 2,7 & 2,7 & 2,4 \\
\hline & \multicolumn{7}{|c|}{ Micronutrientes $\left(\mathrm{mg} \mathrm{kg}^{-1}\right)$} \\
\hline $\mathrm{Fe}$ & $372,6-570,7$ & $396,4-523,5$ & $38,3-46,8$ & $70,0-200,0$ & $1.325,0$ & 300,0 & 157,0 \\
\hline $\mathrm{Mn}$ & $82,6-114,3$ & $75,8-106,8$ & $45,1-59,6$ & $70,0-400,0$ & 250,0 & 217,0 & 145,0 \\
\hline $\mathrm{Zn}$ & $54,8-68,7$ & $54,5-65,1$ & $15,9-18,3$ & $10,0-50,0$ & 40,0 & 29,0 & 21,0 \\
\hline $\mathrm{B}$ & $14,0-18,7$ & $6,8-8,5$ & $6,2-7,7$ & $4,0-25,0$ & 10,0 & 9,0 & 9,0 \\
\hline $\mathrm{Cu}$ & $5,5-7,6$ & $5,1-6,1$ & $6,0-6,6$ & $3,0-25,0$ & 22,0 & 12,0 & 8,0 \\
\hline
\end{tabular}

(1)Época padrão de amostragem, aos 65 DAS, com coleta da folha-bandeira. ${ }^{(2)}$ Amostras em diferentes DAS.

Tabela 3. Normas de diagnose da composição nutricional para amostragens realizadas aos 30, 40 e 60 dias após a semeadura (DAS) de arroz de sequeiro (Oryza sativa), em Vilhena, RO.

\begin{tabular}{lccccccccccc}
\hline Parâmetro & $\mathrm{N}$ & $\mathrm{P}$ & $\mathrm{K}$ & $\mathrm{Ca}$ & $\mathrm{Mg}$ & $\mathrm{Fe}$ & $\mathrm{Mn}$ & $\mathrm{Zn}$ & $\mathrm{B}$ & $\mathrm{Cu}$ & $\mathrm{MS}$ \\
\hline & & & & & & $30 \mathrm{DAS}$ & & & & \\
Média & 3,488 & 0,719 & 3,119 & 0,960 & 0,988 & $-1,032$ & $-2,549$ & $-2,948$ & $-4,292$ & $-5,193$ & 6,729 \\
Desvio-padrão & 0,169 & 0,164 & 0,180 & 0,157 & 0,149 & 0,503 & 0,465 & 0,260 & 0,350 & 0,240 & 0,139 \\
CV (\%) & 4,85 & 22,74 & 5,78 & 16,38 & 14,93 & 48,75 & 18,24 & 8,83 & 8,16 & 4,62 & 2,07 \\
\hline & & & & & & $40 \mathrm{DAS}$ & & & & & \\
Média & 3,295 & 0,679 & 3,125 & 0,990 & 1,195 & $-0,814$ & $-2,475$ & $-2,809$ & $-4,903$ & $-5,175$ & 6,892 \\
Desvio-padrão & 0,108 & 0,144 & 0,176 & 0,130 & 0,138 & 0,365 & 0,475 & 0,231 & 0,418 & 0,169 & 0,112 \\
CV (\%) & 3,27 & 21,20 & 5,63 & 13,12 & 11,57 & 44,84 & 19,17 & 8,23 & 8,53 & 3,27 & 1,62 \\
\hline & & & & & & $65 \mathrm{DAS}$ & & & & & \\
Média & 3,962 & 1,184 & 2,859 & 1,559 & 1,107 & $-2,710$ & $-2,548$ & $-3,610$ & $-4,543$ & $-4,600$ & 7,338 \\
Desvio-padrão & 0,075 & 0,142 & 0,171 & 0,202 & 0,150 & 0,242 & 0,350 & 0,202 & 0,323 & 0,142 & 0,095 \\
CV (\%) & 1,88 & 12,02 & 5,98 & 12,93 & 13,53 & 8,93 & 13,75 & 5,58 & 7,12 & 3,08 & 1,30 \\
\hline
\end{tabular}


$20 \%$ dos casos, respectivamente; Mn, em 31, 14 e $22 \%$ dos casos; Zn, em 19, 14 e 11\% dos casos; B, em 28, 14 e $25 \%$ dos casos; e $\mathrm{Cu}$, em 19, 29 e $25 \%$ dos casos.

Quando os diagnósticos foram realizados com normas $(\mathrm{X})$ obtidas em épocas diferentes das da época de amostragem (Y), a discrepância entre os diagnósticos foi maior: $\mathrm{N}, 100 \%$ em excesso nutricional no caso de
$X=40 \rightarrow Y=65$, e $100 \%$ insuficiente quando ocorreu o contrário $(65 \rightarrow 40) ; \mathrm{Mg}, 100 \%$ em equilíbrio nutricional no caso de $40 \rightarrow 65,65 \rightarrow 30$ e $65 \rightarrow 40 ; \mathrm{Fe}, 100 \%$ das amostras em excesso no caso de $65 \rightarrow 30$ e $65 \rightarrow 40$; Zn e B, $100 \%$ em equilíbrio, no caso de $40 \rightarrow 65$.

As adubações de cobertura com $\mathrm{N}$ e $\mathrm{K}$, utilizadas de acordo com sistema de produção, não foram capazes

Tabela 4. Proporção (\%) de amostras diagnosticadas como nutricionalmente insuficientes, equilibradas ou em excesso, para análises realizadas em diferentes épocas de amostragem, com diferentes épocas de obtenção das normas de diagnose da composição nutricional (CND).

\begin{tabular}{|c|c|c|c|c|c|c|c|c|c|}
\hline \multirow{3}{*}{$\begin{array}{l}\text { Estado } \\
\text { nutricional }\end{array}$} & \multicolumn{9}{|c|}{ Época de obtenção das normas CND } \\
\hline & \multicolumn{3}{|c|}{$30 \mathrm{DAS}^{(1)}$} & \multicolumn{3}{|c|}{$40 \mathrm{DAS}$} & \multicolumn{3}{|c|}{65 DAS } \\
\hline & 30 & 40 & 65 & 30 & 40 & 65 & 30 & 40 & 65 \\
\hline & & & & & $\overrightarrow{\operatorname{rog} \hat{e}}$ & & & & \\
\hline Insuficiente & 25 & 66 & 0 & 0 & 23 & 0 & 94 & 100 & 25 \\
\hline Equilibrado & 58 & 34 & 11 & 31 & 54 & 0 & 6 & 0 & 53 \\
\hline \multirow[t]{2}{*}{ Excesso } & 17 & 0 & 89 & 69 & 23 & 100 & 0 & 0 & 22 \\
\hline & \multicolumn{9}{|c|}{ Fósforo } \\
\hline Insuficiente & 20 & 14 & 0 & 11 & 20 & 0 & 50 & 43 & 17 \\
\hline Equilibrado & 58 & 80 & 25 & 69 & 66 & 28 & 50 & 57 & 64 \\
\hline \multirow[t]{2}{*}{ Excesso } & 22 & 6 & 75 & 20 & 14 & 72 & 0 & 0 & 19 \\
\hline & \multicolumn{9}{|c|}{ Potássio } \\
\hline Insuficiente & 19 & 14 & 11 & 11 & 20 & 8 & 0 & 0 & 19 \\
\hline Equilibrado & 56 & 60 & 89 & 81 & 46 & 92 & 89 & 97 & 50 \\
\hline \multirow[t]{2}{*}{ Excesso } & 25 & 26 & 0 & 8 & 34 & 0 & 11 & 3 & 31 \\
\hline & \multicolumn{9}{|c|}{ Cálcio } \\
\hline Insuficiente & 22 & 11 & 0 & 19 & 23 & 0 & 39 & 14 & 28 \\
\hline Equilibrado & 53 & 71 & 6 & 67 & 54 & 19 & 61 & 86 & 50 \\
\hline \multirow[t]{2}{*}{ Excesso } & 25 & 18 & 94 & 14 & 23 & 81 & 0 & 0 & 22 \\
\hline & \multicolumn{9}{|c|}{ Magnésio } \\
\hline Insuficiente & 22 & 0 & 0 & 61 & 20 & 0 & 0 & 0 & 22 \\
\hline Equilibrado & 44 & 29 & 92 & 39 & 57 & 100 & 100 & 100 & 58 \\
\hline \multirow[t]{2}{*}{ Excesso } & 33 & 71 & 8 & 0 & 23 & 0 & 0 & 0 & 20 \\
\hline & \multicolumn{9}{|c|}{ Ferro } \\
\hline Insuficiente & 31 & 3 & 94 & 42 & 26 & 97 & 0 & 0 & 20 \\
\hline Equilibrado & 42 & 66 & 6 & 42 & 43 & 3 & 0 & 0 & 69 \\
\hline \multirow[t]{2}{*}{ Excesso } & 27 & 31 & 0 & 16 & 32 & 0 & 100 & 100 & 11 \\
\hline & \multicolumn{9}{|c|}{ Manganês } \\
\hline Insuficiente & 31 & 11 & 0 & 17 & 14 & 0 & 3 & 3 & 22 \\
\hline Equilibrado & 44 & 71 & 100 & 75 & 74 & 100 & 97 & 97 & 53 \\
\hline \multirow[t]{2}{*}{ Excesso } & 25 & 18 & 0 & 8 & 12 & 0 & 0 & 0 & 25 \\
\hline & \multicolumn{9}{|c|}{ Zinco } \\
\hline Insuficiente & 19 & 6 & 69 & 31 & 14 & 0 & 0 & 0 & 11 \\
\hline Equilibrado & 58 & 60 & 31 & 64 & 74 & 100 & 47 & 34 & 72 \\
\hline \multirow[t]{2}{*}{ Excesso } & 23 & 34 & 0 & 5 & 12 & 0 & 53 & 66 & 17 \\
\hline & \multicolumn{9}{|c|}{ Boro } \\
\hline Insuficiente & 28 & 77 & 3 & 0 & 14 & 0 & 0 & 3 & 25 \\
\hline Equilibrado & 50 & 23 & 97 & 39 & 66 & 100 & 97 & 97 & 47 \\
\hline \multirow[t]{2}{*}{ Excesso } & 22 & 0 & 0 & 61 & 20 & 0 & 3 & 0 & 28 \\
\hline & \multicolumn{9}{|c|}{ Cobre } \\
\hline Insuficiente & 19 & 6 & 0 & 17 & 29 & 0 & 92 & 71 & 25 \\
\hline Equilibrado & 75 & 83 & 19 & 78 & 57 & 28 & 8 & 29 & 53 \\
\hline Excesso & 6 & 11 & 81 & 5 & 14 & 72 & 0 & 0 & 22 \\
\hline
\end{tabular}

${ }^{(1)} \mathrm{DAS}$, dias após a semeadura. 
de diminuir o número de parcelas consideradas insuficientes em diagnósticos $65 \rightarrow 65$. As adubações de cobertura foram realizadas em épocas críticas de aumento da demanda nutricional pela cultura: no perfilhamento e na diferenciação do primórdio floral (Ferreira \& Santiago, 2012).

$\mathrm{Na}$ avaliação do estado nutricional, considerouse até o terceiro nutriente mais limitante, em todas as combinações $X \rightarrow Y$ (Tabela 5). Quando avaliado com normas específicas para cada época $(\mathrm{X}=\mathrm{Y})$, o $\mathrm{Cu}$ foi identificado como o mais limitante aos 40 e 65 DAS, e o Ca como o segundo mais limitante em todas as épocas. A ordenação do grau de limitação nutricional exercido por cada nutriente é uma das grandes vantagens de métodos derivados do DRIS, como é o caso do método CND.

Apesar de a adubação de cobertura não ter resolvido os casos de insuficiência nutricional de $\mathrm{N}$, ela teve efeito sobre a ordem de limitação nutricional exercida pelo nutriente. $\mathrm{O} \mathrm{N}$ foi o segundo nutriente mais limitante nas avaliações aos 40 DAS (Tabela 5) e deixou de figurar entre os três mais limitantes nas avaliações aos 65 DAS $(\mathrm{X}=\mathrm{Y})$.

Nas avaliações $30 \rightarrow 65$ e $40 \rightarrow 65$, Fe e $\mathrm{Zn}$ foram os nutrientes mais limitantes, respectivamente. $\mathrm{Na}$ avaliação realizada com norma específica $(65 \rightarrow 65)$, porém, esses nutrientes não figuraram entre os mais limitantes. Esse resultado, provavelmente, está relacionado ao fato de a demanda nutricional das culturas variar ao longo de seu desenvolvimento.

Tabela 5. Ordem de deficiência nutricional em arroz de sequeiro (Oryza sativa) em diferentes épocas de amostragem, de acordo com as normas de diagnose da composição nutricional (CND) obtidas para essas épocas.

\begin{tabular}{|c|c|c|c|}
\hline \multirow{2}{*}{$\begin{array}{l}\text { Ordem de } \\
\text { limitação }\end{array}$} & \multicolumn{3}{|c|}{ Normas CND } \\
\hline & $30 \mathrm{DAS}^{(1)}$ & $40 \mathrm{DAS}$ & $65 \mathrm{DAS}$ \\
\hline & \multicolumn{3}{|c|}{ Amostragem aos 30 DAS } \\
\hline 10 & $\mathrm{Mn}$ & $\mathrm{Mg}$ & $\mathrm{N}$ \\
\hline $2^{\circ}$ & $\mathrm{Ca}, \mathrm{Fe}$ e B & $\mathrm{Fe}$ & $\mathrm{Cu}$ \\
\hline \multirow[t]{2}{*}{$\underline{3^{\underline{o}}}$} & - & $\mathrm{Zn}$ & $\mathrm{P}$ \\
\hline & \multicolumn{3}{|c|}{ Amostragem aos 40 DAS } \\
\hline $1 \stackrel{0}{2}$ & $\mathrm{~B}$ & $\mathrm{Cu}$ & $\mathrm{N}$ \\
\hline $2^{\circ}$ & $\mathrm{N}$ & N, P, Ca, Mg, Mn e Zn & $\mathrm{B}$ \\
\hline \multirow[t]{2}{*}{$\underline{3^{\circ}}$} & $\mathrm{P}$ e $\mathrm{K}$ & - & - \\
\hline & \multicolumn{3}{|c|}{ Amostragem aos 60 DAS } \\
\hline $1^{\circ}$ & $\mathrm{Fe}$ & $\mathrm{Fe}$ & $\mathrm{Cu}$ \\
\hline $2^{\circ}$ & $\mathrm{Zn}$ & $\mathrm{Zn}$ & $\mathrm{Ca}$ \\
\hline 3 음 & $\mathrm{K}$ & - & $\mathrm{K}$ e B \\
\hline
\end{tabular}

${ }^{(1)} \mathrm{DAS}$, dias após a semeadura.
No caso de Fe e Zn, por exemplo, as normas obtidas aos 30 e 40 DAS foram derivadas de amostras com maiores concentrações dos nutrientes (Tabela 2) do que as obtidas aos 65 DAS e, portanto, apontaram maior frequência de limitação desses nutrientes.

O P pode ser considerado como o macronutriente mais limitante à produtividade do arroz (Fageria \& Baligar, 1997), e, entre os micronutrientes, apenas a aplicação de $\mathrm{Zn}$ tem resultado em resposta produtiva da cultura (Fageria et al., 2011).

A adubação com nitrogênio favorece principalmente o perfilhamento do arroz (Barreto et al., 2002), enquanto a com fósforo está ligada diretamente ao desenvolvimento da cultura (Rotili et al., 2010) e a de potássio, ao aumento da massa de grãos (Farinelli et al., 2004). Contudo, são poucas as informações sobre os efeitos da adubação com micronutrientes.

Guindani et al. (2009) chamam atenção para uma possível superestimação das deficiências de micronutrientes com o uso do DRIS. Os autores relatam que, embora esses nutrientes tenham sido apontados entre os que apresentaram maior grau de limitação, dificilmente seriam observadas respostas significativas à sua aplicação, em termos da produtividade das culturas. Os micronutrientes apresentam estreito intervalo de disponibilidade entre a deficiência e a toxidez, e requerem cautela na recomendação de adubação (Corrêa et al., 2006). Portanto, apesar da análise no presente trabalho ter indicado o $\mathrm{Cu}$ como o nutriente mais deficiente aos 40 e 65 DAS, não é possível afirmar que a adubação com esse nutriente seria convertida em ganhos significativos na produção.

Como não houve indivíduos com baixos teores foliares de $\mathrm{Fe}$ e $\mathrm{Zn}$ na população de referência das normas obtidas aos 30 e 40 DAS, o uso dessas normas tornou o método muito sensível à diminuição nos teores desses nutrientes pelo efeito de diluição. Da mesma forma, quando se utiliza normas obtidas aos 65 DAS, o método torna-se hipersensível aos maiores teores dos nutrientes observados aos 30 e 40 DAS, o que ficou claro na Tabela 4, em que o Fe foi diagnosticado como em excesso em $100 \%$ dos casos nas avaliações $65 \rightarrow 30$ e $65 \rightarrow 40$.

A concordância entre os diagnósticos realizados com a amostragem antecipada (30 e 40 DAS) com o obtido por meio da amostragem padrão (65 DAS) foi maior para $\mathrm{K}$, exceto quando foram utilizadas três classes de PRA, com a norma de 30 DAS, em que a concordância para $\mathrm{Mg}$ foi maior (Tabela 6). 
Tabela 6. Grau de concordância entre os diagnósticos antecipados, realizados com amostras foliares retiradas aos 30 e 40 dias após a semeadura (DAS), e diagnóstico obtido com amostragem em época padrão, aos 65 DAS, de acordo com dois ou três níveis do potencial de resposta à adubação (PRA), de arroz de sequeiro (Oryza sativa) cultivado em Vilhena, RO.

\begin{tabular}{|c|c|c|c|c|c|c|c|c|c|c|}
\hline \multirow[t]{2}{*}{ Níveis PRA } & \multicolumn{10}{|c|}{ Grau de concordância (\%) } \\
\hline & $\mathrm{N}$ & $\mathrm{P}$ & $\mathrm{K}$ & $\mathrm{Ca}$ & $\mathrm{Mg}$ & $\mathrm{Fe}$ & $\mathrm{Mn}$ & $\mathrm{Zn}$ & B & $\mathrm{Cu}$ \\
\hline & \multicolumn{10}{|c|}{ Amostragem aos 30 DAS } \\
\hline 2 & 75,00 & 83,33 & 91,67 & 72,22 & 77,78 & 25,00 & 77,78 & 41,67 & 77,78 & 75,00 \\
\hline \multirow[t]{2}{*}{3} & 25,00 & 30,56 & 61,11 & 22,22 & 66,67 & 19,44 & 52,78 & 25,00 & 50,00 & 25,00 \\
\hline & \multicolumn{10}{|c|}{ Amostragem aos 40 DAS } \\
\hline 2 & 75,00 & 83,33 & 88,89 & 72,22 & 77,78 & 22,22 & 77,78 & 36,11 & 75,00 & 75,00 \\
\hline 3 & 22,22 & 30,56 & 58,33 & 22,22 & 58,33 & 19,44 & 52,78 & 19,44 & 47,22 & 27,78 \\
\hline
\end{tabular}

De forma geral, as normas antecipadas de 30 e 40 DAS não conseguiram prever satisfatoriamente a classificação quando se utilizou três classes de PRA, com concordância menor ou igual a $50 \%$ para a maioria dos nutrientes (Tabela 6). Para os nutrientes $\mathrm{N}, \mathrm{P}, \mathrm{Ca}, \mathrm{Mn}, \mathrm{B}$ e $\mathrm{Cu}$, o uso de apenas duas classes de PRA aumentou consideravelmente a concordância entre os diagnósticos, para níveis superiores a 75\%, em média. Entretanto, para Fe e Zn, mesmo com o uso de apenas duas classes de PRA, a concordância mantevese baixa.

\section{Conclusões}

1. A antecipação da diagnose foliar em arroz de sequeiro (Oryza sativa) depende do desenvolvimento de normas de diagnose da composição nutricional específicas para o período amostral.

2. O uso de apenas duas classes para a interpretação do potencial de resposta à adubação aumenta a concordância entre os diagnósticos realizados com a amostragem antecipada e a padrão, o que, por sua vez, aumenta a possibilidade de antecipar o período da diagnose foliar, na cultura do arroz.

\section{Agradecimentos}

À Coordenação de Aperfeiçoamento de Pessoal de Nível Superior (Capes), pela concessão de bolsa de Mestrado ao primeiro autor.

\section{Referências}

BARRETO, J.F.; RAMALHO, A.R.; MARTINS, G.C.; UTUMI, M.M.; DIAS, M.C.; XAVIER, J.J.B.N. Recomendações técnicas para o cultivo do arroz no Amazonas. Manaus: Embrapa Amazônia Ocidental, 2002. 11p. (Embrapa Amazônia Ocidental. Circular técnica, 12).

BEAUFILS, E.R. Diagnosis and recommendation integrated system (DRIS): a general scheme of experimentation and calibration based on principles developed from research in plant nutrition. Pietermaritzburg: University of Natal, 1973. 132p.

BELL, P.F.; KOVAR, H.L. Rice. In: CAMPBELL, C.R. (Ed.). Reference sufficiency ranges for plant analysis in the southern region of the United States. Raleigh: North Carolina Department of Agriculture and Consumer Services Agronomic Division, 2009. (Southern Cooperative Series Bulletin, 394).

BOLETIM climatológico de Rondônia: ano 2010. Porto Velho: Coordenadoria de Geociência, Secretaria do Estado do Desenvolvimento Ambiental, 2010.

CARMO, C.A.F. de S.; ARAÚJO, W.S. de; BERNARDI, A.C. de C.; SALDANHA, M.F.C. Métodos de análise de tecidos vegetais utilizados na Embrapa Solos. Rio de Janeiro: Embrapa Solos, 2000. (Circular técnica, 6).

CARVALHO, A.J.C. de; FONTES, P.S.F.; FREITAS, M.S.M.; MONNERAT, P.H.; FONTES, A.G. Yellow passion fruit plant nutritional diagnosis at different phenological stages by the diagnosis and recommendation integrated system method. Journal of Plant Nutrition, v.34, p.614-626, 2011. DOI: 10.1080/01904167.2011.538558.

COMPANHIA NACIONAL DE ABASTECIMENTO. Acompanhamento da safra brasileira - grãos 2013 - quinto levantamento - safra 2013/2014. Brasília: Conab, v.1, n.5, fev. 2014. 69p.

CORRÊA, J.C.; COSTA, A. de M.; CRUSCIOL, C.A.C.; MAUAD, M. Doses de boro e crescimento radicular e da parte aérea de cultivares de arroz de terras altas. Revista Brasileira de Ciência do Solo, v.1, p.1077-1082, 2006. DOI: 10.1590/ S0100-06832006000600017.

COUNCE, P.A.; KEISLING, T.C.; MITCHELL, A.J. A uniform, objective, and adaptive system for expressing rice development. Crop Science, v.40, p.436-443, 2000. DOI: 10.2135/ cropsci2000.402436x. 
FAGERIA, N.K.; BALIGAR, V.C. Upland rice genotypes evaluation for phosphorus use efficiency. Journal of Plant Nutrition, v.20, p.499-509, 1997. DOI: 10.1080/01904169709365270.

FAGERIA, N.K.; BALIGAR, V.C.; JONES, C.A. Growth and mineral nutrition of field crops. New York: CRC Press, 2011. $586 \mathrm{p}$.

FAGERIA, N.K.; BARBOSA FILHO, M.P.; MOREIRA, A.; GUIMARÃES, C.M. Foliar fertilization of crop plants. Journal of Plant Nutrition, v.32, p.1044-1064, 2009. DOI: 10.1080/01904160902872826.

FARINELLI, R.; PENARIOL, F.G.; FORNASIERI FILHO, D.; BORDIN, L. Características agronômicas de arroz de terras altas sob plantio direto e adubação nitrogenada e potássica. Revista Brasileira de Ciência do Solo, v.28, p.447-454, 2004. DOI: 10.1590/S0100-06832004000300006.

FERREIRA, C.M.; SANTIAGO, C.M. (Ed.). Informações técnicas sobre o arroz de terras altas: estados de Mato Grosso e Rondônia - safras 2010/2011 e 2011/2012. Santo Antônio de Goiás: Embrapa Arroz e Feijão, 2012. 112p. (Embrapa Arroz e Feijão. Documentos, 268).

GUINDANI, R.H.P.; ANGHINONI, I.; NACHTIGALL, G.R. DRIS na avaliação do estado nutricional do arroz irrigado por inundação. Revista Brasileira de Ciência do Solo, v.33, p.109-118, 2009. DOI: 10.1590/S0100-06832009000100012.

INSTITUTO BRASILEIRO DE GEOGRAFIA E ESTATÍSTICA. Produção agrícola estadual: lavoura temporária 2012. Disponível em: <http://www.ibge.gov.br/estadosat>. Acesso em: 7 mar. 2013.

KURIHARA, C.H. Demanda de nutrientes pela soja e diagnose de seu estado nutricional. 2004. 114p. Tese (Doutorado) Universidade Federal de Viçosa, Viçosa.

MACEDO, M.C.M. Integração lavoura e pecuária: o estado da arte e inovações tecnológicas. Revista Brasileira de Zootecnia, v.38, p.133-146, 2009. DOI: 10.1590/S1516-35982009001300015.

MARSCHNER, P. (Ed.) Mineral nutrition of higher plants. $3^{\text {rd }}$ ed. New York: Elsevier, 2012.

PARENT, L.É. Diagnosis of the nutrient compositional space of fruit crops. Revista Brasileira de Fruticultura, v.33, p.321-334, 2011. DOI: 10.1590/S0100-29452011000100041.
PARENT, L.E.; DAFIR, M. A theoretical concept of compositional nutrient diagnosis. Journal of the American Society for Horticultural Science, v.117, p.239-242, 1992.

PARTELLI, F.L.; VIEIRA, H.D.; CARVALHO, V.B. de; MOURÃO FILHO, F. deA.A. Diagnosis and recommendation integrated system norms, sufficiency range, and nutritional evaluation of Arabian Coffee in two sampling periods. Journal of Plant Nutrition, v.30, p.1651-1667, 2007. DOI: 10.1080/01904160701615525.

PRADO, R. de M. Nutrição de plantas. São Paulo: Ed. da UNESP, 2008. 407p.

RODRÍGUEZ, O.; RODRÍGUEZ, V. Desarrollo, determinación e interpretación de normas DRIS para el diagnóstico nutricional en plantas. Una revisión. Revista de la Facultad de Agronomía (LUZ), v.17, p.449-470, 2000.

ROTILI, E.A.; FIDELIS, R.R.; SANTOS, M.M. dos; BARROS, H.B.; PINTO, L.C. Eficiência do uso e resposta à aplicação de fósforo de cultivares de arroz em solos de terras altas. Bragantia, v.69, p.705-710, 2010. DOI: 10.1590/S0006-87052010000300023.

SUMNER, M.E. Applications of Beaufils' diagnostic indices to maize data published in the literature irrespective of age and conditions. Plant and Soil, v.46, p.359-369, 1977. DOI: 10.1007/ BF00010092.

SUMNER, M.E.; BEAUFILS, E.R. Diagnosis of the N, P and K requirements of sugarcane irrespective of plant age and season using Beaufils' system (DRIS): preliminary observations. Proceedings of the South Africa Sugar Technologists' Association, v.49, p.137-141, 1975.

WADT, P.G.S. Relationships between soil class and nutritional status of coffee plantations. Revista Brasileira de Ciência do Solo, v.29, p.227-234, 2005. DOI: 10.1590/S0100-06832005000200008.

WADT, P.G.S.; ALVAREZ V., V.H. Monitoramento nutricional. In: WADT, P.G.S. (Org.). Manejo do solo e recomendação de adubação para o Estado do Acre. Rio Branco: Embrapa Acre, 2005. p.283-304.

WADT, P.G.S.; ANGHINONI, I.; GUINDANI, R.H.P.; LIMA, A.S.T. de; PUGA, A.P.; SILVA, G.S. da; PRADO, R. de M. Padrões nutricionais para lavouras arrozeiras irrigadas por inundação pelos métodos da CDN e chance matemática. Revista Brasileira de Ciência do Solo, v.37, p.145-156, 2013. DOI: 10.1590/ S0100-06832013000100015.

Recebido em 7 de maio de 2014 e aprovado em 23 de fevereiro de 2015

Pesq. agropec. bras., Brasília, v.50, n.3, p.250-258, mar. 2015

DOI: 10.1590/S0100-204X2015000300009 